\title{
Chapter 6 \\ The FOREVER-DO Game: A Big Data Fishing Expedition
}

\author{
Jill Townsley and Carlo Ferigato
}

\begin{abstract}
The FOREVER-DO Game is a participatory visual artwork, that takes the form of a relational game and installation. It is one outcome from an art science collaboration supported by the European Commission's Joint Research Centre in Italy. The artwork was developed under the theme of 'Big Data and Identity' it considers the causal chain that our individual and social actions may have as they flow across the interface of the human and the digital network. The work focuses on data flow that has some correspondence with coordination and communication, as identified by Petri Nets theory. Carl Adam Petri introduced the Nets in the 1970s, they are one of the techniques used today for the analysis of Process Data. When data is ordered in time, the causal chains of data are collected in 'processes'. One simple representation of a process net is the 'bucket chain' used to represent two fundamental aspects of data flow: selection and transfer. By using the bucket chain as a platform this project is focussed on a discipline of data where some 'causal flow', possibly represented by nets, has a role in the data analysis game. The resulting artwork encourages the viewer/participant to flow through an installation following a set of rules on a box, their actions coordinate according to the incidence of chance, selection, transfer and the similarity or difference of everyday objects.
\end{abstract}

Keywords Petri Nets $\cdot$ Coordinated behaviours $\cdot$ Data $\cdot$ Flow $\cdot$ Communication discipline $\cdot$ System $\cdot$ Time $\cdot$ Space $\cdot$ Repetition $\cdot$ Difference $\cdot$ Unfolding $\cdot$

Folding $\cdot$ Selection and transfer $\cdot$ Participation

J. Townsley $(\varangle)$

University of Huddersfield, Huddersfield, UK

e-mail: jill@jilltownsley.com

C. Ferigato

European Commission, Joint Research Centre (JRC), Ispra, Italy

e-mail: carlo.ferigato@ec.europa.eu

(C) The Author(s) 2020

R. Earnshaw et al. (eds.), Technology, Design and the Arts-Opportunities and Challenges, Springer Series on Cultural Computing, https://doi.org/10.1007/978-3-030-42097-0_6 


\subsection{Introduction}

Artistic activity is a game, whose forms, patterns and functions develop and evolve according to periods and social contexts; it is not an immutable essence [1].

The FOREVER-DO Game is a socially relational public artwork or happening, in which people flow around a space, moving between a network of coloured doormats, according to a set of instructions printed on a box. This seemingly absurd game activates interactions between players, who meet randomly in space and time. The coordinated nature of the rules-based system dictates the path that the players travel, it is in itself a socially related construct. A construct that rationalises data flow, making visible the hidden nature of all data flowing within our big data systems. The work presents us with a symbiotic relationship between scientific theory and art practice, while also raising questions about socially mediated, rules-based interactions, especially those that cross the physical and digital interface.

The game is positioned within today's digitally networked age, it mirrors the theoretical construct of a 'Bucket Chain' network, a simple form of Petri Net system, proposed by the computer scientist Carl Adam Petri in the 1970s. In theoretical Computer Science, nets are instruments used for the analysis and design of systems, distributed in time and space. The strength of these nets is their explicit representation of fundamental situations of coordination and concurrency among system agents. Agents can be anything: objects, computers and/or human beings.

The first manifestation of this work was played/exhibited during the Milan Digital Week in March 2019, in the Palazzo dei Giureconsulti, near the Duomo, in collaboration with the MC3 research group on concurrent systems at the University of Milan Bicocca. The game was repeated in October 2019 for the Resonance III festival, at the European Commission's Joint Research Centre (JRC) in Italy, this time with additional digital visualisations developed through the application of Radio Frequency Identification tracking (RFID). Trackers were placed inside each of the 2,000 boxes used to play the game, the feedback from the trackers was detected by antennas with the data then displayed digitally alongside the installation (Fig. 6.1). It is the first presentation of the FOREVER-DO Game in Milan that we discuss here.

The cross-disciplinary collaboration between artist Jill Townsley and computer scientist Carlo Ferigato was important to the way that the artwork has developed. The work was commissioned by SciArt, an art science organisation embedded within the JRC. The JRC is the European Commission's science and knowledge service which employs scientists to carry out research in order to provide independent scientific advice and support to EU policy. The SciArt mission:

brings together scientists, artists and policy makers to discuss matters of concern from various points of view, not only to the JRC and the European Commission but also more widely to society [2].

Additionally, SciArt set the agenda of 'big data and identity' as the theme for the collaboration, stating: 


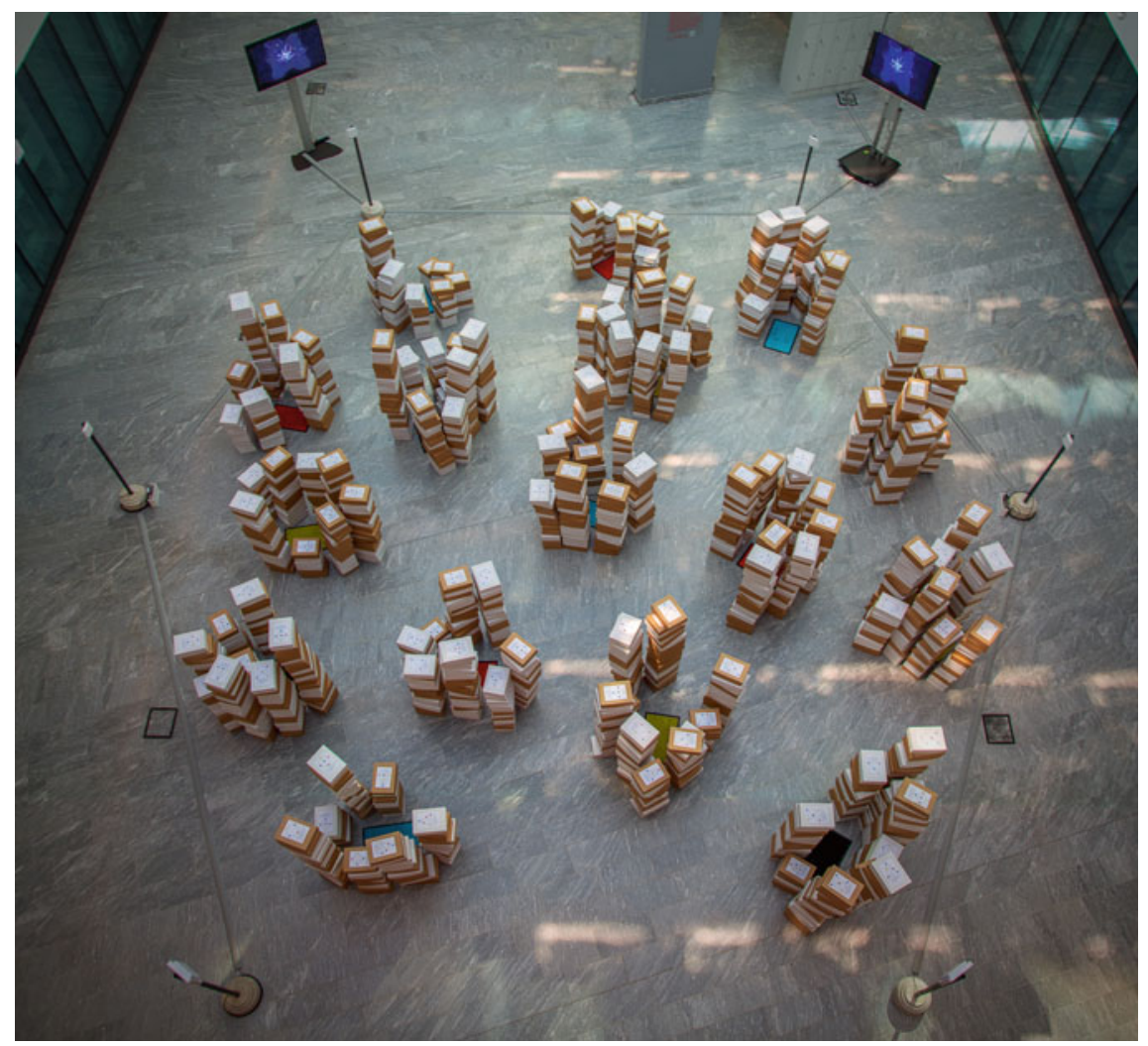

Fig. 6.1 The FOREVER-DO Game (2019 October JRC exhibition) Copyright @ J. Townsley 2019

Big Data have erupted in our daily lives and we want to join the discussion on how to turn Big Data into culture, not leaving it only in the hands of technicians, trolls and corporations. We want to become hackers of our own future. We think it is time we make them ours, these Big Data, this digital transformation, in a vast collective effort open to all [2].

To do this SciArt have proposed a new concept 'DATAMI':

We see the datami as a virtual tatami [a Japanese mat], made of the data that we cherish, our data and those of our families and friends, our discoveries and curiosities, our roaming and conversations, all of these making up our new identities [3].

This is the theme for the Resonances III Festival and Exhibition held at the JRC in October 2019, and it is within this overarching context that the FOREVER-DO project was formed (Fig. 6.2).

In order to help convey the significance of the FOREVER-DO Game we look to the work of art critic and curator Nicolas Bourriaud and his concept of relational aesthetics. This supports the identification of some formal qualities in the artwork especially the interpersonal cooperation that is present within the FOREVER-DO Game and the significance of this model of interaction as a creative tool, to critically reflect on 


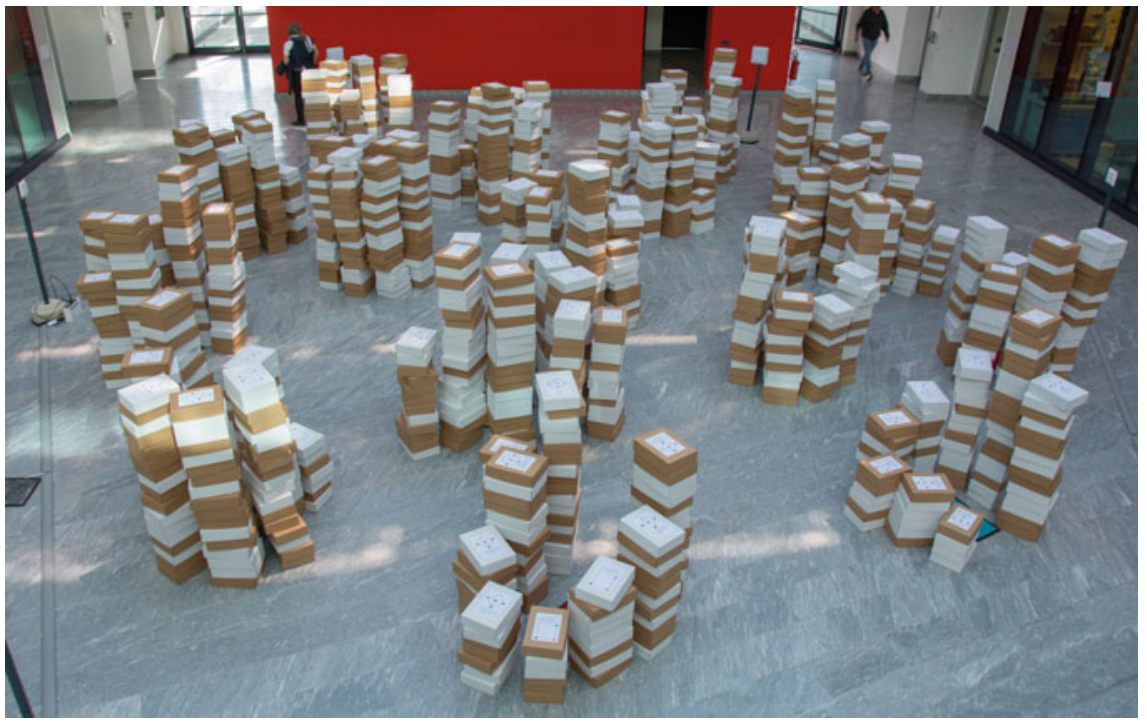

Fig. 6.2 The FOREVER-DO Game (2019 October JRC exhibition) Copyright $\odot$ J. Townsley 2019

society. We also look to the media theorist, critic and philosopher Boris Groys and his writing on 'flow'. The flow of people and objects within the game is important and Groys helps identify how flow may define moments of temporary happening. Happenings that can have some governance over social interaction, presenting as 'an event not as a thing' [4]. Groys's theory helps us draw some conclusions about the way that the game mediates the viewer/participant in order to highlight the interface of the digital and physical realm, especially in relation to big data operations and to net theory.

Finally, to help us consider how the artworks analogue process interacts with the human digital interface we will be looking not only to Petri Nets theory, (as the inspiration for the way that the game has been developed), but also reading Carl Adam Petri's writing as a critical tool. Looking specifically at his Communication Disciplines delivered as a set of twelve conceptual tools. He proposed them in a lecture in 1976 to support reasoning in communication systems design, and suggested that they could help build the ideal computer system [5]. A system that could enhance human cooperation and overcome some of the many problems he foresaw for a computerised world going forward. Petri's principal aim was to help realise a computer system whose applications could support humanity to develop its best possible future. It is this call from the 1970s that dovetails so significantly with the concept of DATAMI proposed by the SciArt team and motivates the creation of the FOREVER-DO project. 


\subsection{A Fishing Expedition-the Method}

Meeting within the context of the JRC, and in response to the SciArt theme of Big Data and identity, we (artist Jill Townsley and computer scientist/researcher Carlo Ferigato) realised that 'process' held an important position within both of our working practices. Ferigato's research around the process design of communication systems, specifically systems identified by Petri Nets theory, and Townsley's artwork emphasising the process of the art object, definitive beyond subject and object. The process presented us with common ground to articulate our practices in language that cut across our respective fields, producing a rich dialogue and exchange of creative thinking. It identified important key words beyond the process itself, such as system, time, space, flow, coordinated behaviour, repetition, difference, unfolding, folding, selection and transfer.

Through analysing our processes, nets became a key link between the art and science used in our collaboration. Put poetically, we became fishermen casting our nets, to fish across disciplines into the sea of big data sets in order to extract coherent patterns and procedures. We found patterns pertinent to Petri Nets theory, that could be visualised as art. From this, we have developed two symbiotic artworks the FOREVER-DO Game and the FOREVER-DO Infestation.

While we consider only the FOREVER-DO Game here, it is important to know that the placement of boxes within the game became data that has driven another artwork, the FOREVER-DO Infestation. The data, gathered from one piece of work flows on to inform the realisation of another. In this way, authorship is shared with every person who has played the game by placing a box on a pile.

While the FOREVER-DO Project as a whole (the Game and Infestation) explores the idea of 'fishing' into data sets, the nets we use are multifariously allegorical. Beyond being a fisherman's tool, the word 'net' has reference to our digital networks, the Internet. Nets are also the title given to the formal computer science tools 'Petri Nets' (as outlined in the introduction). One of Petri's examples, the 'Bucket Chain' is the main source of inspiration for the FOREVER-DO Project. It is outlined in some detail in the theoretical context below but is an explanation of the mechanics of the FOREVER-DO Game.

\subsection{The FOREVER-DO Game- the First 'Catch'}

This artwork propels its human participants on a physical journey that mirrors a 'causal flow' of data (Fig. 6.3). The basic idea of causal flow is to understand how factors influence one another. The game seeks to envision the invisible influence between human action and data. As the game is played, a sculptural installation consisting of towers of boxes develops in the gallery space. These box-towers simulate a data pile, presenting a physical object that records the coordinated meetings of each individual who (following a set of instructions on the box) encounters another 


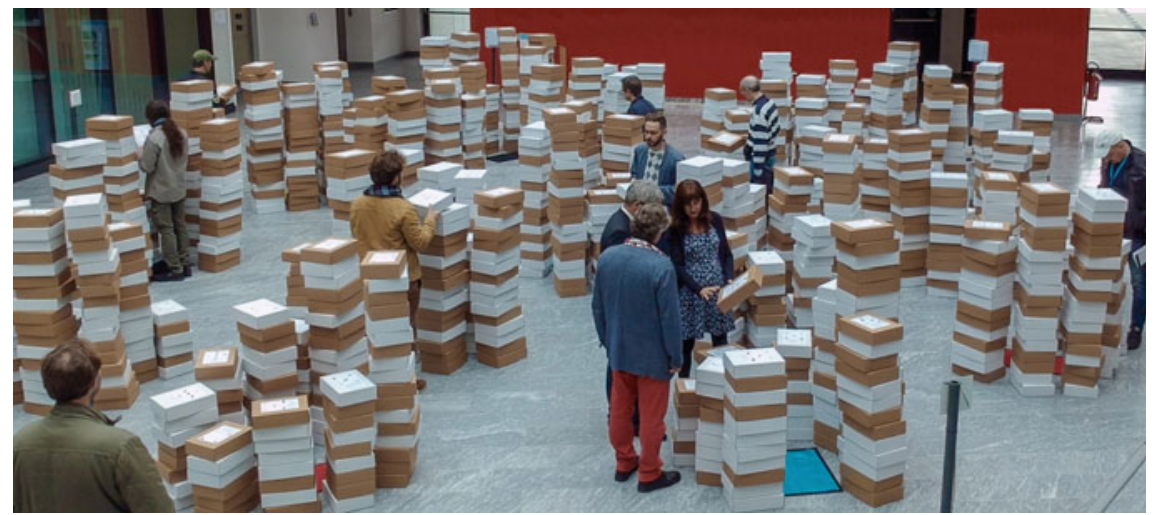

Fig. 6.3 The FOREVER-DO Game (2019 October JRC exhibition) Copyright $\odot$ J. Townsley 2019, included by permission

player at a coloured mat and finds (through random chance) that their boxes contain identical objects.

The mechanics of the game are as follows. In a large room there are placed a set of objects:

- Sixteen coloured doormats, placed randomly around the room, specifically,

- 4 Yellow,

- 4 Red,

- 4 Blue and

- 4 Black doormats.

- 2,000 boxes (made to hold a ream of A4 paper).

- 1,000 brown boxes-randomly dispersed through the installation,

- 1,000 white boxes - randomly dispersed through the installation.

- Each box has instructions (the rules of the game) printed onto the top.

- There are 6 sets of rules offering slight variations.

- The 6 sets of rules are randomly distributed (shuffled) throughout the 2000 boxes.

- Each box contains (randomly) one item of mass-produced wooden cutlery:

- a knife,

- fork or,

- spoon.

With this absurd combination of objects, the game is ready to be played.

Visitors to the exhibition are invited to pick up a box and follow the instructions printed on the top (Fig. 6.4). The instructions send people on a journey travelling 
Fig. 6.4 Boxes and Instructions Copyright $\odot \mathrm{J}$.

Townsley 2019

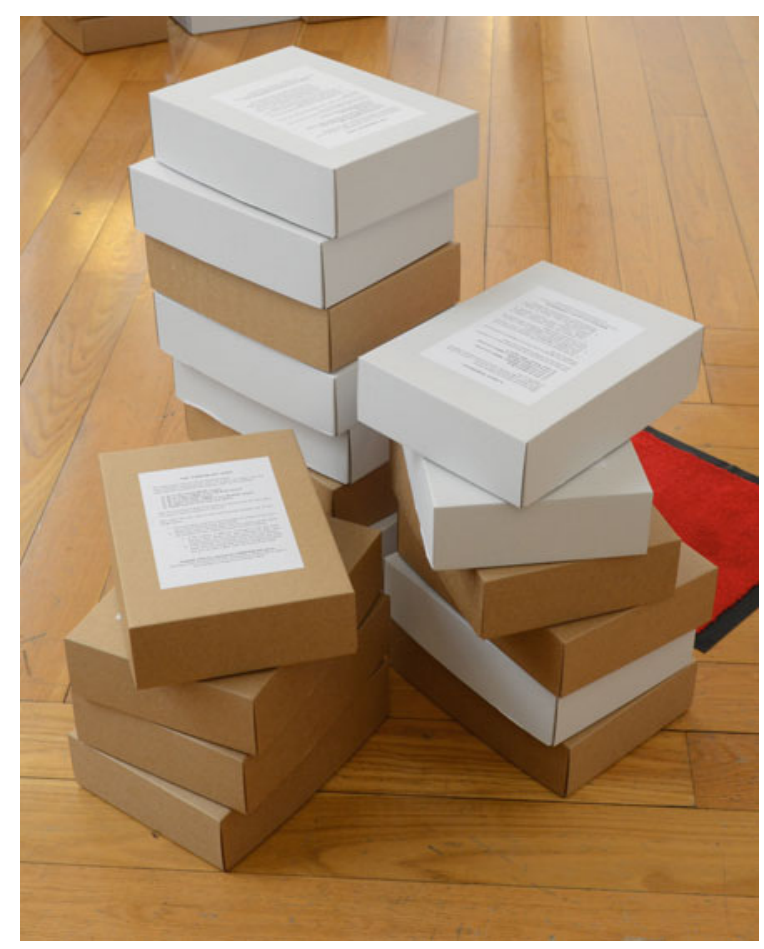

through the space from coloured mat to coloured mat. There are 6 sets of instructions, all presenting a different order of coloured mats to visit:

(1) go to the closest BLUE mat;

(2) go to either a RED or to a BLACK mat;

(3) go to another mat that is the same colour as your present one;

(4) go to any YELLOW mat;

(5) repeat forever from rule one above.

Players physically 'flow' through the space of the gallery, moving according to the rules on their box. The brown or white boxes contain an everyday object: a knife, a fork or a spoon. When individuals meet at a mat, they open the box to compare the contents. Depending on the 'local' circumstance around the similarity or difference of those contents, players encounter one of two consequences:

1. They continue to flow around the game:

- this happens when their compared objects are different (non-repeated) - then the objects are exchanged and the flow continues according to the rules.

2. They stop and exit the game:

- if the objects are the same (repeated) then players are asked to leave their box and object behind, placed on a tower at the point of coordination. 


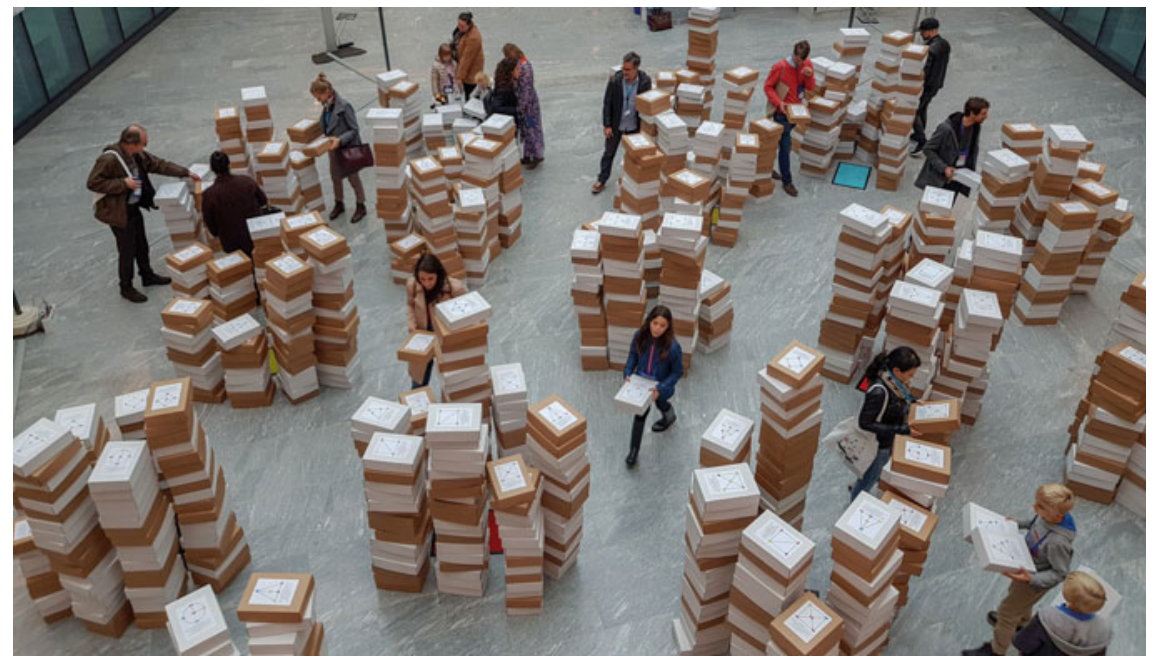

Fig. 6.5 Players of the game Copyright (c) J. Townsley 2019, photograph Pierre-Stuart Rostain, included by permission

In this way a 'causal flow' happens, when non-repeated coordination occurs, objects are exchanged and continue their journey, independent of player or boxflowing on, to continue the FOREVER-DO. Alternatively, when repeated coordination occurs (people meeting with the same objects), the flow is halted and boxes build up around the mats. Over time the number of boxes in the towers gather randomly around the mats, infesting the space and forming a sculptural installation (Fig. 6.5).

The installation makes visible a poetic data trail of coordinated human interactions, recording the incidence of two people meeting at a mat, to find that through random chance, their boxes contain identical objects. The coloured doormats act like nodes in a giant network. Receiving the flow of information as people and boxes move from mat to mat, enabling coordination and a place for human interaction (Fig. 6.6).

As the box-towers grow, data may be extracted from the game in many different ways. The piles of white and brown boxes could be interpreted as binary code. The number of boxes clustered around a coloured doormat also give that mat an integer value. From this data, new digital, conceptual and physical constructs may be developed. Reorganised data is used to form a new sculpture: the FOREVER-DO Infestation. Alternatively, a new event or happening could be derived as presented in the FOREVER-DO Game 2. In other words, a new fishing expedition can begin (Figs. 6.7 and 6.8).

More generally, the gathering, reorganisation and extraction of information, as presented in the FOREVER-DO Game, is indicative of the way data flows today. Data (collected in a myriad of ways) flows forever through our digital systems. Systems and data unimaginably vast in form, yet invisible in time and space. This data can be organised and extracted in whatever way society chooses: it can be reorganised and 


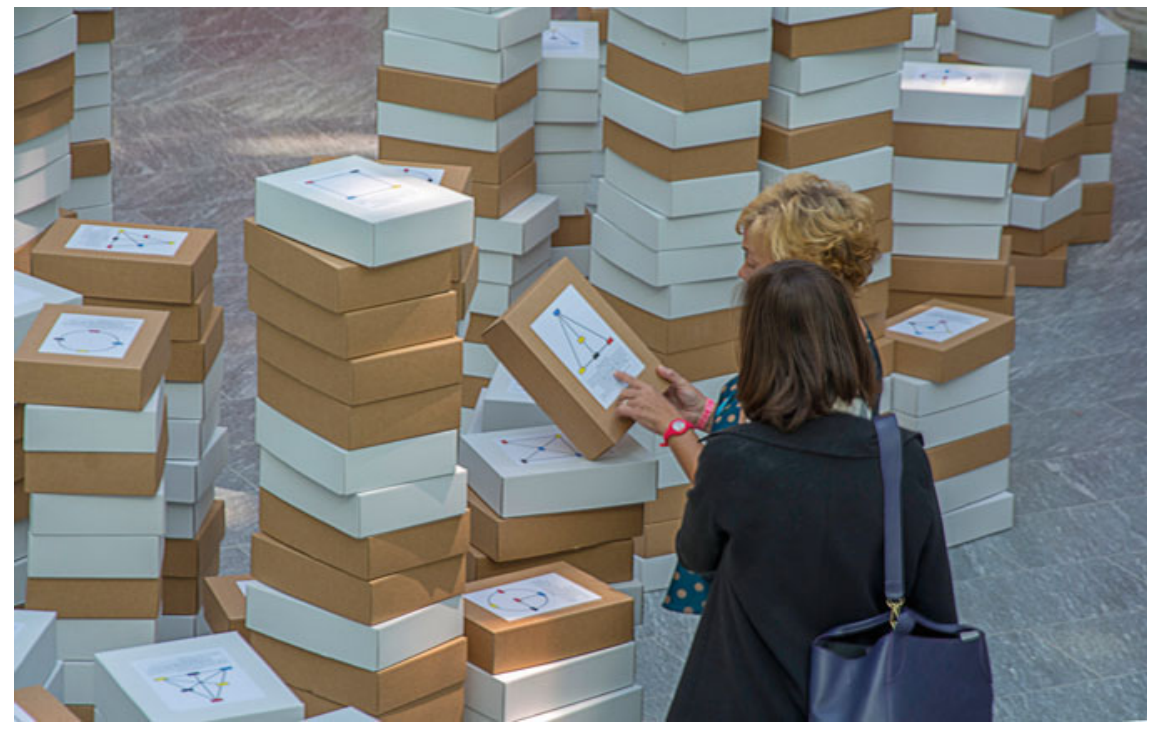

Fig. 6.6 Players of the game and the box-towers Copyright (C) J. Townsley 2019 included by permission

extracted to help us do great things for humanity, such as understand diseases; or it can be used less responsibly, to smartly target us to purchase more mass-produced goods.

\subsection{Petri Nets, Communication and the Bucket Chain}

The structure of the FOREVER-DO Game takes its principles from Petri Nets theory, in experiential and analogue terms it makes visible a form of communication that is happening across the interface of our physical and digital worlds. The installation presents a data trail made from the flow of people around a room, flowing until random instances of coordination happen between players. This has resonance with Petri Nets theory, in its desire to rationalise information flow as a resource in communication.

The history of Petri Nets can be traced back to the Ph.D. thesis of Carl Adam Petri in 1962 [6]. His aim was to establish a new theory of communication-based on two assumptions:

(a) there exists an upper limit on the speed of signals;

(b) there exists an upper limit on the density with which information can be stored.

These assumptions can be considered as natural ones today, but they were not so natural in the 1960s, when the theory of abstract computing machines (automata) was 
Fig. 6.7 Image of towers around a mat Copyright $(\mathcal{O} \mathrm{J}$. Townsley 2019 included by permission

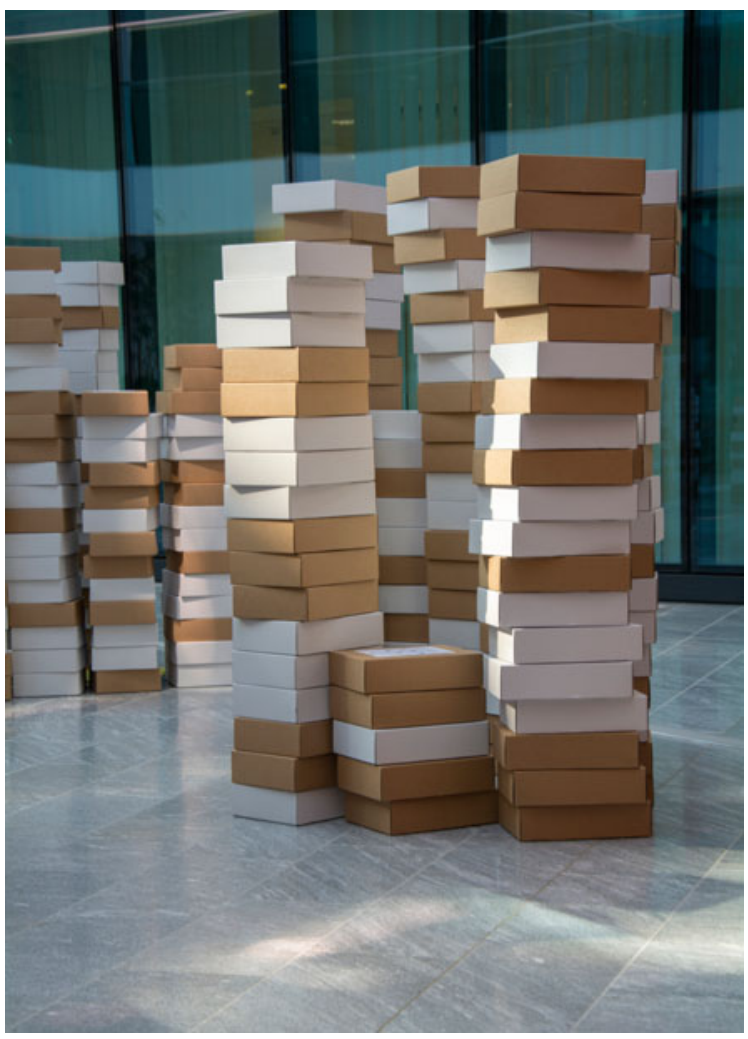

developing rapidly and, sometimes without regard for its potential limits in actual hardware realisations.

As a consequence of these natural assumptions, Petri analysed the subject of 'information flow' in a new way, based on the relations between the structure and behaviour of automata and on the role of information as a resource in communication. In his view, communication becomes an organised activity involving automata and people. Communication with automata, the title of the thesis, refers precisely to this organised activity where 'with' has the double meaning of 'between automata and people' and 'using automata as communication medium'. Considering automata as organised communication mediums, as we do today through the Internet for example, Petri opened the way to new applications of the discipline 'pragmatics', as a branch of linguistics, in view of a modern theory of communication systems [7].

Petri's bucket chain is a simple example of net theory and one way of rationalising data flow. It presents a scenario of coordinated behaviour between firemen extinguishing a fire. Figure 6.9 shows figures coordinating a task to carry water from a tank to a fire, using a chain of buckets.

The sequence in Fig. 6.10 explains how the coordination of behaviour (the figures) and the flow of data (the water from the tank to fire) operate. The theoretical net 


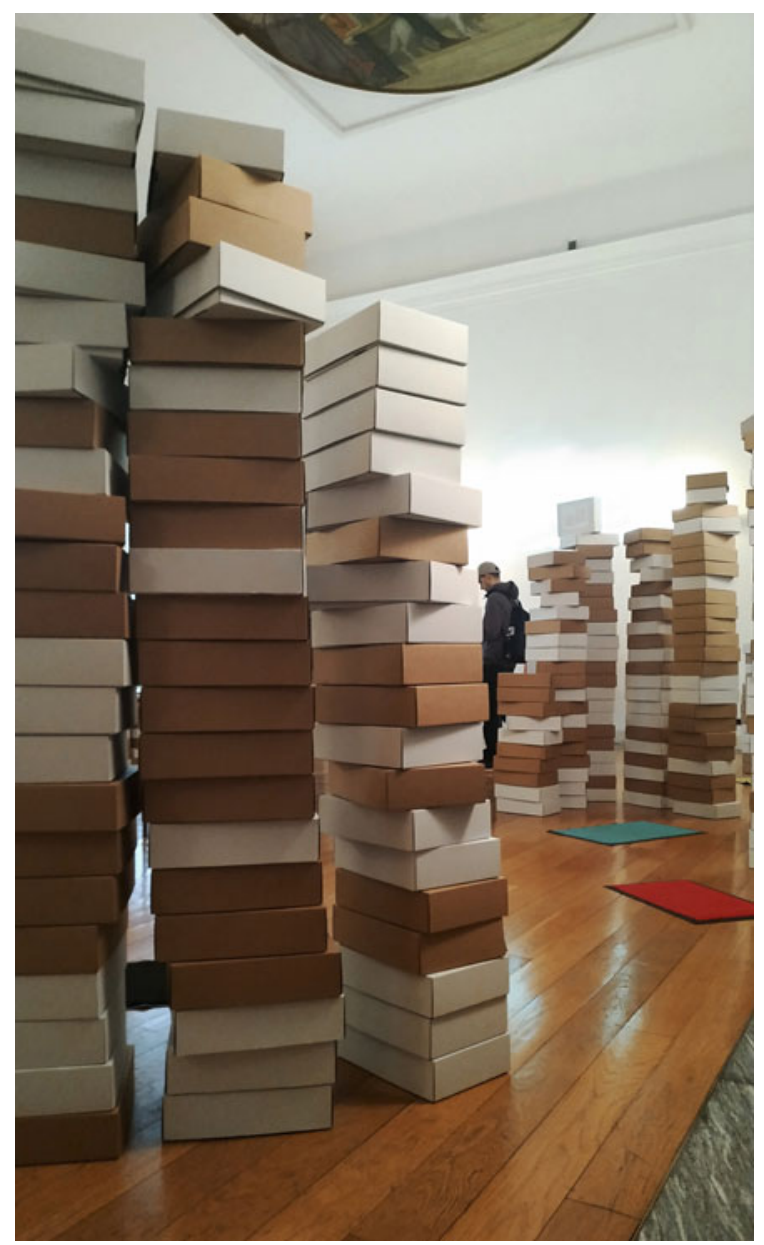

Fig. 6.8 Image of towers around a mat Copyright ( ) J. Townsley 2019 included by permission

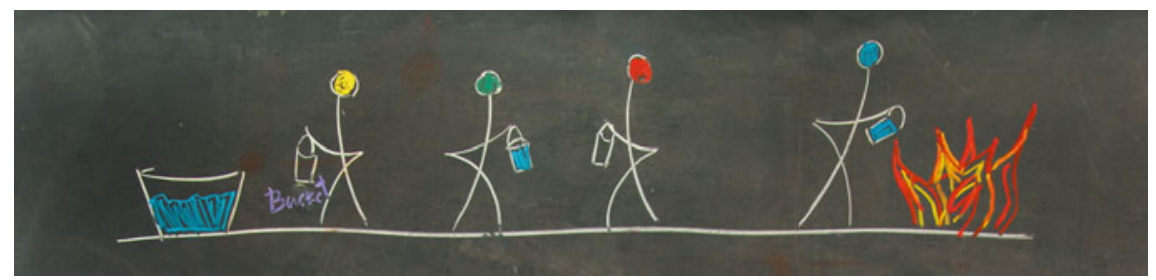

Fig. 6.9 The Bucket Chain (drawing from Ferigato's blackboard, 2019) Copyright $\odot$ J. Townsley 2019 


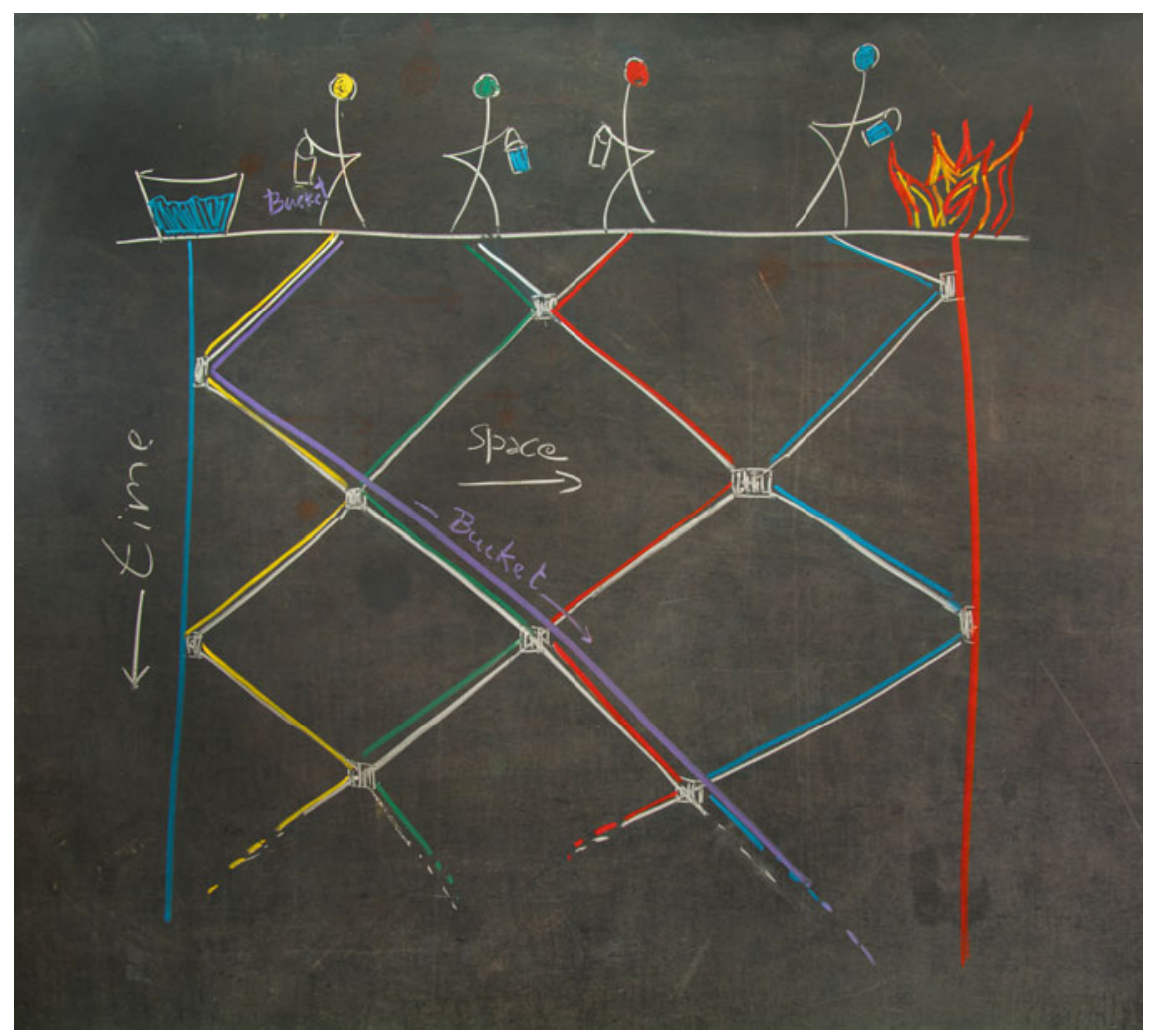

Fig. 6.10 The Bucket Chain and its unfolding in space and time (Ferigato 2018) Copyright $@$ J. Townsley 2019

'unfolds' through time and space to illustrate how the coordination of the figures putting out the fire operates. This is Petri's clever graphical way of explaining simple net theory through the bucket chain scenario.

As the information, in this case, the water in bucket B, flows smoothly from the water tank to fire, it is also possible to see how each fireman moves left and right until they meet to exchange the buckets (Fig. 6.11).

The proposition: 'A walks in the direction right' can be represented, following the formalism of Petri's Occurrence Nets, by a circle, representing in an abstract space the persistence of the proposition.

Importantly, the truth (or value) of the proposition 'A walks in the direction right' is changed by the occurrence of events. For example, as in Fig. 6.10, two men walking in opposite directions along the same line will eventually meet at points in space represented as squares in Fig. 6.11. This 'meeting event' changes the respective directions of the two men, as if they were bouncing balls. So, we can represent a chain of firemen extinguishing a fire by carrying back and forth buckets of water in an abstract way, as in Fig. 6.11. 
Fig. 6.11 The bucket chain as a net (Ferigato 2018) Copyright $(\subset)$ J. Townsley 2019

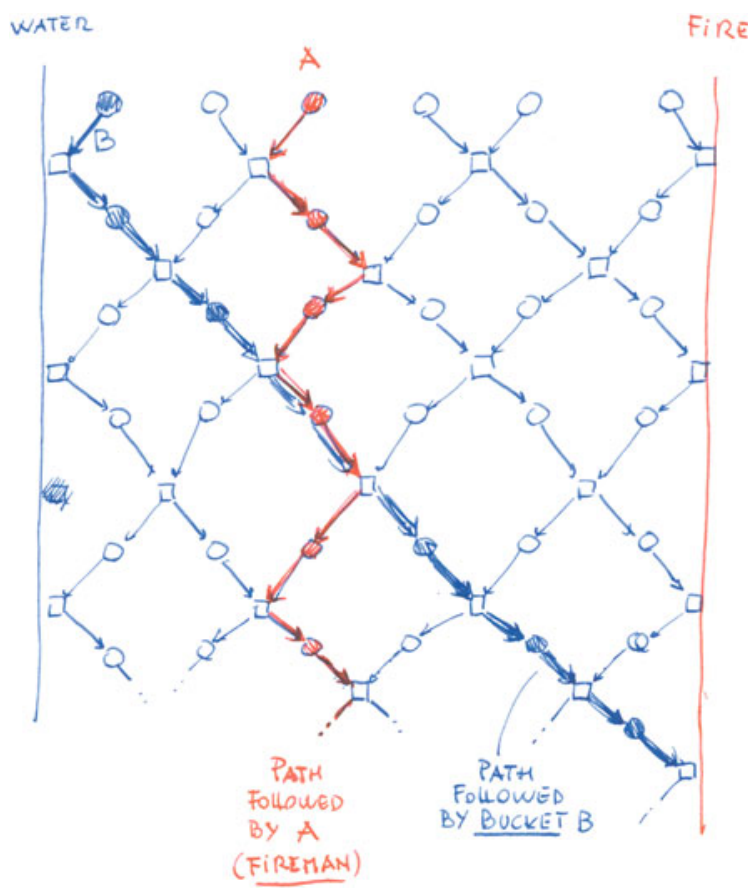

The net presents events, the meetings between two firemen, or, at the edge between the first fireman on the left and the reservoir of water and the last fireman on the right and the fire. Note that meetings between firemen do not happen in exactly the same place, nor are they regulated by any definite intervals of time.

This is the principle illustrated in the game, people flowing from coloured mat to coloured mat according to a set of rules on a box. They are moving through the space as if in a deep fog, meeting other players outside of a determinate system of time and space. The rules written on the box propel the player according to a networked system, but when another player is encountered, the meeting event happens outside of the rule of a clock or defined by a specific determination of place. Also, the proposition for flow is changed with each encounter as objects are exchanged. In our digital world information also flows forever, it can be extracted at any time or place that interaction happens. This model also presents the basic principle of artificial intelligence, where the information has the ability to change according to an occurrence or meeting event. 


\subsection{Theoretical Context: Design and Analysis of (Coordinated) Communication Systems}

computer technology supplies us not [just] with a medium for artificial intelligence nor with a machine which may be used solely for computation, but with a medium for communication and for strictly organised information flow, a medium which may induce major changes in the modalities of co-operation between human beings [5].

The contemporary designer of communication systems is forced, in his profession, to consider relations between computers and society that are not yet consolidated in a unique practice or competence. Along the history of design of communication systems, plain consideration of measurable components like memory, throughput and availability, has shown its weakness in respect to less measurable subjects like delegation of tasks to computers, keeping control on copies of data and identify data as belonging to a given category.

Petri proposed the first set of twelve communication disciplines that are today still a valid starting point for designing and analysing communication systems. He addressed the design of communication systems from a wide perspective, as coordinated activities involving humans and computers.

The 'Communication Disciplines' were presented in September 1976 during a lecture given at Newcastle University and later published in 'Computer Systems Design: Proceedings of the Joint IBM-University of Newcastle upon Tyne Seminar (B. Shaw editor). He stated:

I tried to classify practical problems from my long list, according to my stated viewpoints of the role the computer [5].

His categories were:

1. Synchronisation

2. Identification

3. Copying

4. Addressing

5. Naming

6. Cancellation

7. Composition

8. Modelling

9. Authorization

10. Valuation

11. Delegation

12. Reorganisation

His principal aim was to look into the future of computer systems and their applications, in order to support humanity to develop towards a brighter future. His conclusion was that computer system design should ideally be developed to 'organise information flow' in order to support 'cooperation between human beings'. As the true maverick he was, Petri proposed a list that he hoped computer systems developers 
could potentially use to 'concentrate our attention on' building a computer system that would enhance human cooperation and solve some of the problems he foresaw for a computerised world in the future.

\subsection{Fishing the Net of the Communication Disciplines}

To identify some of the processes and functions operating within the FOREVER-DO Game, we will use a selection of the 12 Communication Disciplines (highlighted in bold) as critical tools. In doing so we are also contextualising the game within a broader critical framework.

Petri states that the discipline of identification 'covers the question of pattern recognition' [5]. The installation is a result of pattern recognition, specifically, a record of all the players whose synchronised meetings resulted in the identification of copied objects. Identifying sets of objects also indicates a destination at a mat. This is most obvious as the composition of stacked boxes grows around the mats in the room. Petri proposes that composition 'is concerned with determining the structure of documents relative to a material or conceptual carrier' [5]. In traditional art terms, there is a strong correlation here between 'function and form' in material composition. The towers are subject to the rules of the game, the rules act as a 'conceptual carrier' determining the number of boxes in a pile. This takes the form of an ever-emerging flow of data, offered through time, as boxes move around the installation.

\footnotetext{
Contemporary art escapes the present not by resisting the flow of time but by collaborating with it. It produces artistic events, performances, temporary exhibitions that demonstrate the transitory character of the present order of things and the rules that govern contemporary social behaviour. Imitation of the anticipated future, may manifest itself only as an event not as a thing [4].
}

Groys's observation is a good way of thinking about the game, especially in the many ways flow can be identified. Flow happens in the reorganisation that ensures the slow evolution of the installation. Petri talks about reorganisation in relation to mechanised tasks within a nuclear power plant, he highlights the potentially 'disastrous' consequences if the flow of tasks and information are not adequately reorganised to meet changing situations. In the game, reorganisation is the process of becoming, an ever-changing contingent object. Reorganisation is embedded within the flow of the game and also enforced by external contingencies.

The idea of artwork adapting to contingent conditions is not new, artwork can exist for just a moment within a definitive contingent space, temporary artwork such as: Allan Kaprow's happenings and environments, or the installations of Doris Salcedo, Olafur Eliasson and teamLab et al. Martha Buskirk considers 'The Contingent Object of Contemporary Art' in her book by the same name [8]. She refers to the artwork Gnaw (1992) by Janine Antoni where the artist chews, over many days, two 600pound cube's, one made of chocolate, the other of lard. The action of gnawing results in a constant reorganisation. Buskirk says: 
Thus the experience of the work, including the relationship of its components to one another, changes depending on when and in what part of the cycle one sees it, and also diverges from photographic records of its appearance [8].

This is pertinent to the FOREVER-DO, not only from the position of a passive viewer, but also for the player who is not only witnessing the reorganisation of boxes in a room but accepting active agency within that reorganisation. This implicates the player as an author within the ongoing realisation of the game. Petri says of authorization:

this discipline is concerned not only assigning and schematically representing access rights but also with scheduling obligations [5].

By thinking about authorization in this way, a parallel can be drawn between authorization within a system, such as Petri indicates, and authorship commonly placed within art production, the 'authorial' process of an artwork. If, within the FOREVER-DO Game, authorship is shared across participants, objects, systems rules and networks, it cannot be solely located. Rather, obligations are shared, there is no single owner or receiver. Petri died in 2010 yet his inspiration is still implicit in the game, an authorial obligation through time. Authorial agency is delegated across all the agents of the game, flowing across the human, object and conceptual realm.

In this way Delegation is forming an ever-changing collaboration between the mechanics of the game and the society of people who participate in it, Petri identifies in his networks a 'delegation of tasks from one agency to another' [5].

This shared social context is fluid, outside of a singular vision. There is, however, a fixed position in the naming of the groups of objects: a knife; a fork; a spoon. 'Naming is understood as denoting objects structures' [5, p. 177]. Naming-becomes the subject of coordination, the similarity or difference, triggering the selection or transfer and the cancellation of the player in the game. As players track objects in the game they also have an experience of a route travelled and can anticipate a future route to travel. The pattern of flow through the network is in this way an experiential one, as infinite possibilities for coordinated behaviour are generated through random instances.

The synchronisation is 'partially' controlled by the system mechanism, which embraces chance, ensuring that synchronicity happens outside of a deterministic time or place. In this way, the game is mirroring Petri's premise that synchronisation is based on the 'partial ordering in terms of causality as opposed to an ordering to time' [5]. This mirrors Groys's observation on flow being collaborative of time rather than fixing it [4].

Value, such as it is in FOREVER-DO, resides in the moment of participation or observation, not in a fixed object. Petri's consideration of valuation states: 'the instant the information is registered by the observer it already loses its value because it may not be presented anew.' [5]. With this definition, valuation offers a very tricky problem to the traditions of an art market. Traditionally value in art has been placed on the object but, in the game, creative value is in the performative relational aesthetic of the work; it is essentially objectless, a process rather than an object. In this way, value 
is transient, unable to be 'presented anew' a data value, that can only be experienced within the moment.

In an attempt to contextualise art made in today's global context, the French curator Nicholas Bourriaud coined an art movement, the Altermodern. He defined it as a moment when it is possible to produce something:

from a vision of human history as constituted of multiple temporalities ... a positive vision of chaos and complexity [1].

In the Tate triennial exhibition by the same name, 'Altermodern' (2009). The triennial presented artworks from artists such as Marcus Coats, Mathew Darbysher and Franz Ackerman whose artworks offered a document, narrative or engagement with change. Earlier in his book 'Relational Aesthetics' (1998), he offers examples of art practice as a game:

Artistic activity is a game, whose forms, patterns and functions develop and evolve according to periods and social contexts; it is not an immutable essence [1].

Art practice, based on the relational form, is presented in FOREVER-DO as a meeting point of exchange, a physical human interaction, within a networked system. In our digital age, these exchanges are often an opportunity for data mining. Points, relational in time and space, where data is gathered, producing much of our big data; data that is held outside of our physical realm.

The role of artwork is no longer to form imaginary and utopian realities, but to actually be ways of living and models of action within the existing real, whatever the scale is chosen by the artist [1].

The FOREVER-DO Game also represents a 'model of action within the existing real'. Big data, whose functions control us beyond our everyday perception, is here and is real. And yet, the otherness of the nature of big data, its invisible procedures, make it hard for us to perceive our own individual relationship with it —our essential role within the phenomena of big data systems. Without us, our relational interactions, big data would not exist while at the same time human life is becoming more dependent on the information big data feeds back to us. In reality, perhaps, we are already cyborgs in a close and symbiotic relationship with big data itself.

The art historian Edward A. Shanken, in his essay Art in the Information Age (2001), considers work by artists such as Hans Haacke and Joseph Kosuth, he says:

'meaning and value are not embedded in objects, institutions, or individuals so much as they are abstracted in the production, manipulation and distribution of signs and information' [9].

The process of the FOREVER-DO offers a distribution of signs and information, through the search for repetition. Repetition here is the key to unlocking the FOREVER-DO Game. It is the copying of objects (two knives, forks or spoons) that signifies the end of the player's participation, while the process of the game continues. Copying for Petri is a 'message-occurrence in a definite pragmatic context' [5]. In the game, this is a process held within an unfolding never-ending chain of events, Nietzsche like in its eternal return. In 'Thus Spake Zarathustra' (1917) Nietzsche 
describes two eternal paths 'This long lane backwards: it continueth for eternity. And that long lane forward-that is another eternity' [10]. Most importantly the paths come together at a gateway: 'The name of the gateway is inscribed above: This Moment' [10].

In the game, the most significant 'moments' are the coordinated meetings accumulating to model the towers of boxes. Petri utilises the discipline of modelling to guard against rigidity of implementation of established systems across different fields. He states:

our 'models of thinking', tend to gain some illegitimate independence once they have proved successful on a particular field, and are then - per analogy but without care - transferred to other fields [5].

Perhaps this is a warning against the transformative analysis between Petri's disciplines (as pertinent to computer systems) and the FOERVER-DO Game. However, the very temporality of the FOREVER-DO Game can perhaps embody modelling, where:

we are able to deal with mathematical models in which notions of a temporal ordering is replaced by that of an ordering in terms of causality [5].

Whether you are an active participant in the game or a viewer on the periphery of the game, you are in communication with the model of the game and its objects. This is not a fixed exchange but a communication flow (a causality), with all agents causally influencing one another in a relational sense.

Nicolas Bourriard draws on such symbiotic forms when he proposes that through the act of 'inclusion' - the art object is causally significant to the participation of the viewer. Indicating that there is an energy exchange between the art object and the participant.

The game is a destination in flux, a place directed by the rules, e.g. ' $g$ o to the closest blue mat'. Petri offers a fluidity of destination with his discipline of addressing: ' $b y$ this we mean the description of routes or systems of paths through the net of channels and agencies' [5]. This flow of address as a destination, is an agency of the ever becoming. Addressing within the flow of the game drives it on to new beginnings, this chimes with Groys when he says:

Being immersed in the flow of things, one cannot return to previous moments in time or experience the events of the past [4].

Addressing also happens in a subtler way too, as social address between players in the game. The form of address has a systemic origin beyond the game, residing in the social etiquette of human behaviour. This presents us with a wider network of behaviours and systems. Petri has proposed that his net systems could have a role in conflict resolution. He suggests, nets could help reconcile complex human problems, emotionally driven problems, beyond the mechanical, systematic or digital, by offering clear alternative channels of communication. 


\subsection{Conclusion}

Art does not predict the future, but rather demonstrate the transitory character of the present - and thus open the way for the new [4].

By thinking about art as a process or event, as relational, societal and social, a new place is offered for authorship and realisation. This is not only the post-modern reform of authorship, deferred or shared, but authorship within the moment of a contingent ever flowing process - the FOREVER-DO.

This conceptual space made real through event also requires a temporal place. For the FOREVER-DO Game the place of the Palazzo Giureconsulti at Duomo in central Milan, and temporarily occupied by Milan digital week, is important. Milan Digital Week is itself a social construct, a temporal event into which interested parties' (visitors and participants) occupy space temporarily. Visitors are in this way already complicit within the flow of events.

The historically embedded definitions of art and art practice, particularly the traditional consideration of value in relation to object, are being challenged through utilising digital systems as integral tools. By visualising, in analogue terms, this relationship between the participant (the player of the game) and the system (the rules and network of the game), the FOREVER-DO highlights our individual complicity within the greater whole. Places of meeting within the game indicated by simple coloured doormats are also nodes within a networked system. When individuals meet through a flow of random incidents, objects are either exchanged or offloaded onto the data pile. The piles of boxes record the incidents of two humans meeting and randomly possessing a repeated object. This process presents a digital and analogue description of events through time and offers a visualisation of a similar scientific modelling that the Petri Nets identify.

Petri's Communication Disciplines, written in the 1970s still 'concentrate our attention on' building a computer system to enhance human cooperation and overcome some of the many problems brought about by our human digital interface. Problems for culture arising from irresponsible systems, technicians, trolls and corporations. We are all complicit in society's broader data communications. As Petri outlined, we must all consider the relations between computers and society that are not yet consolidated in a unique practice or competence. The FOREVER-DO opens a discussion about the interactive trail we leave behind and the systems acting upon us. By reclaiming our digital identity, being aware of the symbiotic relation of self to our larger networks, maybe we can hack our future in a positive way, for the benefit of all.

Acknowledgements The FOREVER-DO project and its resulting artwork (including the FOREVER-DO Game) is a collaboration between Jill Townsley (University of Huddersfield) and Carlo Ferigato (Joint Research Centre of the European Commission-JRC-Ispra, Italy). The project is supported by advice from the MC3 research group at the Milano-Bicocca University and Otolab, Milano. The project is funded by the EU Commission through its SciArt initiative. 


\section{References}

1. Bourriaud, N.: Relational Aesthetics. Les Presses Du Reel (1998)

2. Joint Research Centre (JRC). Available: https://resonances.jrc.ec.europa.eu/. Accessed Oct 2019

3. Joint Research Centre (JRC). Available: https://resonances.jrc.ec.europa.eu/datami. Accessed Oct 2019

4. Groys, B.: In the Flow, Verso Books. London (2018)

5. Petri, C.A: Communication disciplines. In: Shaw, B. (ed.) Computing System Design: Proceedings of the Joint IBM-University of Newcastle upon Tyne Seminar, September 1976, pp. 171-183. University of Newcastle upon Tyne (1977)

6. Petri, C.A.: Kommunikation mit Automaten, Dissertation, Schriften des IIM 2, RheinischWestfälisches Institut für Instrumentelle Mathematik an der Universität Bonn. (Eng. transl. Communication with Automata, Technical report RADC-TR-65-377, Volume I, Final Report, Supplement I) (1962)

7. Bernardinello, L., Cardone, F., Pomello, L.: L'eredit'a di Carl Adam Petri: dagli automi alla comunicazione, Mondo Digitale, febbraio, p. 175 (2016)

8. Buskirk, M.: The Contingent Object of Contemporary Art. The MIT Press, Cambridge, MA, USA (2003)

9. Shanken, E.A.: Art in the Information Age in LEONARDO, vol. 35, No. 4, pp. 433-438 (2002)

10. Nietzsche, F.W.: Thus Spoke Zarathustra. Modern Library, New York (1917)

Open Access This chapter is licensed under the terms of the Creative Commons Attribution 4.0 International License (http://creativecommons.org/licenses/by/4.0/), which permits use, sharing, adaptation, distribution and reproduction in any medium or format, as long as you give appropriate credit to the original author(s) and the source, provide a link to the Creative Commons license and indicate if changes were made.

The images or other third party material in this chapter are included in the chapter's Creative Commons license, unless indicated otherwise in a credit line to the material. If material is not included in the chapter's Creative Commons license and your intended use is not permitted by statutory regulation or exceeds the permitted use, you will need to obtain permission directly from the copyright holder. 